Article

\title{
Synthesis and Biological Evaluation of Novel $N$-Methyl-picolinamide-4-thiol Derivatives as Potential Antitumor Agents
}

\author{
Ting-Ting Huang, Yun-Chuang Huang, Xiao-Yu Qing, Yong Xia, Xun Luo, Ting-Hong Ye \\ and Luo-Ting Yu *
}

State Key Laboratory of Biotherapy, West China Hospital, West China Medical School, Sichuan University, Chengdu 610041, China; E-Mails: huangtingting5400@qq.com (T.-T.H.); hyc159@gmail.com (Y.-C.H.); qingxiaoyu5126@163.com (X.-Y.Q.); zhangweihuascu@163.com (Y.X.); luoxun_cn@126.com (X.L.); yetinghong0908@163.com (T.-H.Y.)

* Author to whom correspondence should be addressed; E-Mail: yuluot@scu.edu.cn;

Tel.: +86-28-8516-4063; Fax: +86-28-8516-4060.

Received: 16 March 2012; in revised form: 3 May 2012 / Accepted: 17 May 2012 /

Published: 25 May 2012

\begin{abstract}
A novel series of $N$-methylpicolinamide-4-thiol derivatives were synthesized and evaluated on human cancer cell lines. Among them, compound $\mathbf{6 p}$ displayed potent and broad-spectrum anti-proliferative activities in vitro on some human cancer cell lines, even better than sorafenib. The advanced kinase inhibitory assays showed that compound 6p could selectively inhibit Aurora-B kinase. The biological results were rationalized by the molecular docking study, which indicated the stable interactions of $\mathbf{6 p}$ with the Aurora-B kinase.
\end{abstract}

Keywords: $N$-methylpicolinamide-4-thiol derivatives; antitumor; Aurora-B kinase; docking study

\section{Introduction}

Aurora proteins-A, -B, and -C, a small family of serine/threonine kinases [1], play distinct roles in the regulation of mitosis [2]. Aurora-A and -B are known to be frequently overexpressed in a wide range of different human tumors, including breast, colon, lung, ovarian, and pancreatic cancers [3-6], suggesting their potential role in tumorigenesis [7]. 
In recent years, the Aurora proteins have been actively pursued as anticancer targets for the discovery of new cancer chemotherapeutics. As a result, several small-molecule inhibitors of the Aurora kinases have been identified, some of which have reached clinical evaluation, including MK-0457(VX-680) [8,9], MLN8054 [10], PHA-739358 [11] and AZD-1152 [12] (Figure 1). However, the ideal inhibitor profile for therapeutic use is still unclear, and these inhibitors with complex structures are difficult to synthesize.

Figure 1. Inhibitors of Aurora kinases.
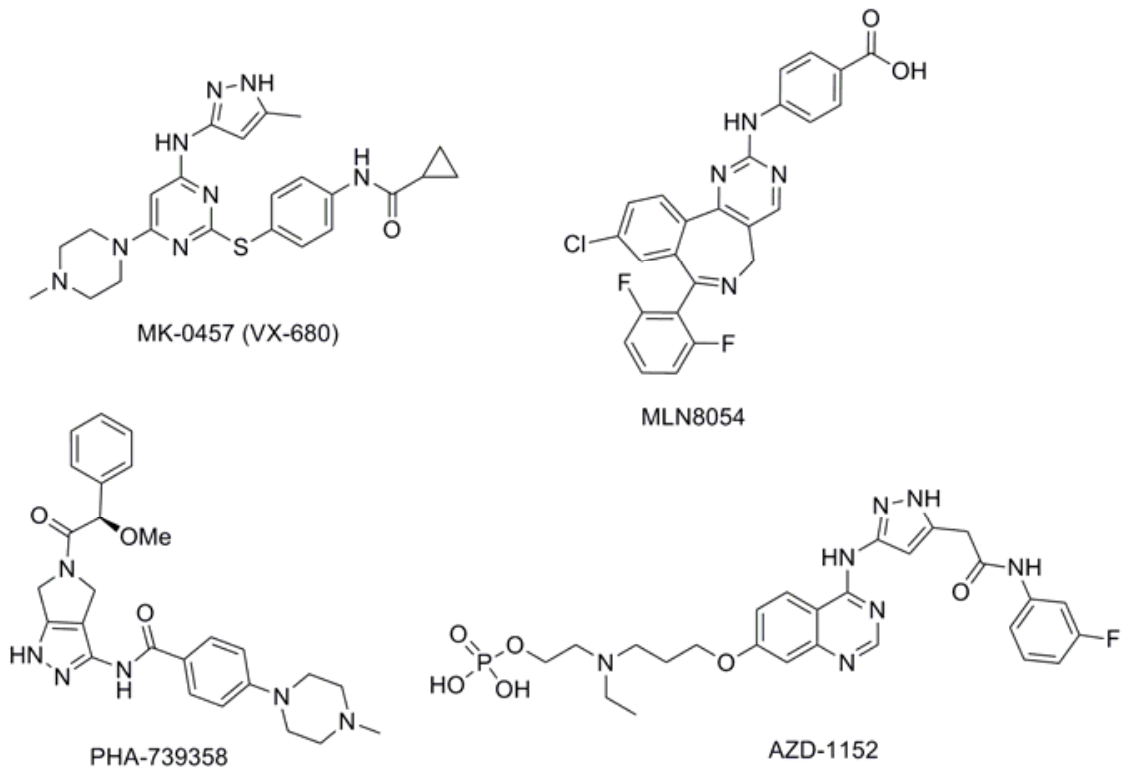

Our group has been interested in the design, screening, synthesis and biological evaluation of novel tumor growth inhibitors. In a previous cell-based screening of our privileged small molecule library, we found that a drug-like compound, $N$-methylpicolinamide-4-thiol (Figure 2), exhibited moderate in vitro cytotoxicity against human hepatocellular carcinoma cell line HepG2 $\left(\mathrm{IC}_{50}=62.96 \mu \mathrm{M}\right)$. In order to find more potent antiproliferative compounds, we designed and synthesized a series of novel $\mathrm{N}$-methylpicolinamide-4-thiol derivatives based on compound 1, employing the structure-activity relationship (SAR) study.

Figure 2. Structure of compound 1.<smiles>CNC(=O)c1cc(Sc2ccc(NC(C)=O)cc2)ccn1</smiles>

1

\section{Results and Discussion}

\subsection{Chemistry}

The synthetic route for the target compounds $\mathbf{1}$ and $\mathbf{6 a - w}$ is shown in Scheme 1. Compound $\mathbf{3}$ was synthesized according to the reported method with a small change [13]. Treatment of 2-picolinic acid (2) 
with $\mathrm{SOCl}_{2}$ in the presence of $\mathrm{NaBr}$ and chlorobenzene afforded acid chloride $\mathbf{3}$ as the corresponding $\mathrm{HCl}$ salt. This $\mathrm{HCl}$ salt was then treated with methylamine solution $(2.0 \mathrm{~mol} / \mathrm{L})$ in methanol to yield 4 . To obtain 5 [14], compound 4 and potassium carbonate were treated with a solution of 4-aminothiophenol, which had been stirred at room temperature for $3.5 \mathrm{~h}$ in the presence of potassium tert-butoxide in dry $N, N$-dimethylformamide. The contents were then heated to $85{ }^{\circ} \mathrm{C}$ under argon for $15 \mathrm{~h}$. Acylation of the amino group of $\mathbf{5}$ with different substituted benzoyl chlorides or alkyl acyl chlorides yielded the target compounds $\mathbf{1}$ and $\mathbf{6 a}-\mathbf{w}$ [15]. The structures of compounds $\mathbf{1}$ and $\mathbf{6 a - w}$ were fully characterized by ${ }^{1} \mathrm{H}-\mathrm{NMR},{ }^{13} \mathrm{C}-\mathrm{NMR}$ and ESI-MS analysis.

Scheme 1. Synthetic route to compounds $\mathbf{1}$ and $\mathbf{6 a}-\mathbf{w}$.
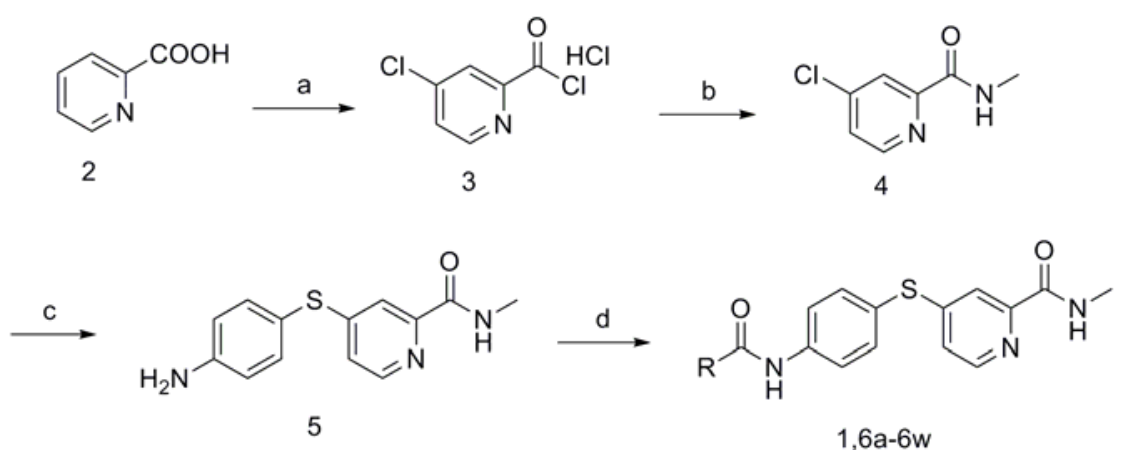

Reagents and conditions: (a) $\mathrm{NaBr}, \mathrm{PhCl}, 50{ }^{\circ} \mathrm{C}$, then $\mathrm{SOCl}_{2}, 19 \mathrm{~h}, 85^{\circ} \mathrm{C}$; (b) $\mathrm{CH}_{3} \mathrm{NH}_{2}, \mathrm{MeOH}$; (c) 4-aminothiophenol, t-BuOK, DMF, $3.5 \mathrm{~h}, 25^{\circ} \mathrm{C}$, then $\mathrm{K}_{2} \mathrm{CO}_{3}, \mathrm{~N}_{2}, 15 \mathrm{~h}, 85^{\circ} \mathrm{C}$; (d) substituted benzoyl acyl chloride or alkyl acyl chloride, $\mathrm{K}_{2} \mathrm{CO}_{3}$, THF, $2 \mathrm{~h}, 0-5^{\circ} \mathrm{C}$.

\subsection{Biological Evaluation}

As shown in Table 1, twenty-three $N$-methylpicolinamide-4-thiol derivatives were synthesized to survey the SAR by evaluating the cell growth inhibitory activity in human liver hepatocellular carcinoma (HepG2) cells. Sorafenib (Figure 3), which was found to significantly prolong the survival of advanced hepatocellular carcinoma (HCC) patients, was selected as the positive control.

Just as seen in the phenyl series 6a-o, unsubstituted phenyl analog 6a showed good inhibitory activity, $\mathrm{IC}_{50}=16.54 \mu \mathrm{M}$. As for the methoxy group, the activity of the analogue with methoxyl at meta-position $\left(\mathbf{6 b}, \mathrm{IC}_{50}=15.43 \mu \mathrm{M}\right)$ is better than that of the para- $(\mathbf{6 c})$ and ortho- $\left(\mathbf{6 d}, \mathrm{IC}_{50}=23.91 \mu \mathrm{M}\right)$ substituted analogues. Compound $6 \mathbf{6 e}\left(\mathrm{IC}_{50}=7.12 \mu \mathrm{M}\right)$, with two methoxy groups substituted at the meta-position, was one of the most potent inhibitors in this series. In terms of halogen atoms (compounds $\mathbf{6 f}-\mathbf{j}$ ), the location had little effect on the activities. However, when the number of the halogen atoms was two, the activity of $6 \mathbf{h}\left(\mathrm{IC}_{50}=10.55 \mu \mathrm{M}\right)$ and $\mathbf{6 i}$ improved greatly. Introduction of the electron-withdrawing groups $\mathrm{CF}_{3}\left[\mathbf{6 k}\left(\mathrm{IC}_{50}=17.04 \mu \mathrm{M}\right), \mathbf{6 l}\left(\mathrm{IC}_{50}=10.96 \mu \mathrm{M}\right), \mathbf{6 m}\right]$ and $\mathrm{NO}_{2}[\mathbf{6 n}$ $\left(\mathrm{IC}_{50}=19.12 \mu \mathrm{M}\right), 6 \mathbf{6 o}$ on the phenyl ring at the meta- and para-position was tolerated.

Replacement of the benzyl group with an aliphatic group was also tolerated. With the $\mathrm{IC}_{50}$ value of $2.23 \mu \mathrm{M}$, compound $\mathbf{6 p}$, which exhibited a nearly 15-fold improvement in inhibitory activity against HepG2 cells over that of sorafenib $\left(\mathrm{IC}_{50}=16.30 \mu \mathrm{M}\right)$, was the most potent analogue in this series.

The number of chloride atom and the length of the carbon chain had a great effect on the activities. The activity was lost when the carbon chain was extended to chloropropyl or butyl chloride structures (compounds $\mathbf{6 s , \mathbf { t }}$ ). The introduction of two or three chloride atoms (compounds $\mathbf{6 q}, \mathbf{r}$ ) also had a 
detrimental effect on the potency. In addition, unsubstituted aliphatic derivatives $\mathbf{6 u}-\mathbf{w}$ were also tested, but their activities were not notable.

Table 1. Inhibition of cell proliferation by compounds $\mathbf{6 a}-\mathbf{w}$.<smiles>[R]C(=O)Nc1ccc(Sc2ccnc(C(=O)NC)c2)cc1</smiles>

$6 a-6 w$

\begin{tabular}{|c|c|c|c|}
\hline \multirow{2}{*}{ Compound } & \multirow{2}{*}{$\mathbf{R}$} & $\mathrm{IC}_{50}(\mu \mathrm{M})^{\mathrm{a}}$ & \multirow{2}{*}{ Structure of the compound } \\
\hline & & HepG2 & \\
\hline $\mathbf{6 a}$ & -phenyl & 16.54 & \\
\hline $6 b$ & -phenyl- $m-\mathrm{OCH}_{3}$ & 15.43 & \\
\hline $6 c$ & -phenyl-p-OCH${ }_{3}$ & 46.97 & \\
\hline 6d & -phenyl-o- $\mathrm{OCH}_{3}$ & 23.91 & \\
\hline $6 e$ & -phenyl-3,5-Di-OCH & 7.12 & \\
\hline $6 f$ & -phenyl-o-Cl & 61.55 & \\
\hline $6 \mathrm{~g}$ & -phenyl- $m$-F & 48.93 & \\
\hline
\end{tabular}


Table 1. Cont.

\begin{tabular}{|c|c|c|c|}
\hline Compound & $\mathbf{R}$ & $\mathrm{IC}_{50}(\boldsymbol{\mu M})^{\mathrm{a}}$ & Structure of the compound \\
\hline $6 h$ & -phenyl-2,4-Di-Cl & 10.55 & \\
\hline $6 \mathbf{i}$ & -phenyl-2,6-Di-F & 23.25 & \\
\hline $6 \mathbf{j}$ & -phenyl-2,3,4,5-Tetra-F & 54.24 & \\
\hline $6 \mathbf{k}$ & -phenyl- $m-\mathrm{CF}_{3}$ & 17.04 & \\
\hline 61 & -phenyl- $p-\mathrm{CF}_{3}$ & 10.96 & \\
\hline $6 \mathrm{~m}$ & -phenyl-o- $\mathrm{CF}_{3}$ & 65.38 & \\
\hline $6 n$ & -phenyl- $m-\mathrm{NO}_{2}$ & 19.12 & \\
\hline 60 & -phenyl-p- $\mathrm{NO}_{2}$ & 33.67 & \\
\hline $6 p$ & $-\mathrm{CH}_{2} \mathrm{Cl}$ & 2.23 & \\
\hline
\end{tabular}


Table 1. Cont.

\begin{tabular}{|c|c|c|c|}
\hline \multirow{2}{*}{ Compound } & \multirow{2}{*}{$\mathbf{R}$} & $\mathrm{IC}_{50}(\mu \mathrm{M})^{\mathrm{a}}$ & \multirow{2}{*}{ Structure of the compound } \\
\hline & & HepG2 & \\
\hline $6 \mathbf{q}$ & $-\mathrm{CHCl}_{2}$ & 44.09 & \\
\hline $6 \mathbf{r}$ & $-\mathrm{CCl}_{3}$ & 94.55 & \\
\hline $6 s$ & $-\mathrm{CH}_{2} \mathrm{CH}_{2} \mathrm{Cl}$ & 70.09 & \\
\hline $6 t$ & $-\mathrm{CH}_{2} \mathrm{CH}_{2} \mathrm{CH}_{2} \mathrm{Cl}$ & 81.53 & \\
\hline $6 u$ & $-\mathrm{CH}_{2} \mathrm{CH}_{3}$ & 75.54 & \\
\hline $6 v$ & $-\mathrm{CH}_{2} \mathrm{CH}_{2} \mathrm{CH}_{3}$ & 180.31 & \\
\hline $6 w$ & $-\mathrm{C}\left(\mathrm{CH}_{3}\right)_{3}$ & 41.15 & \\
\hline Sorafenib & & 16.30 & \\
\hline
\end{tabular}

Figure 3. Structure of Sorafenib.<smiles>CNC(=O)c1cc(Oc2ccc(NC(=O)Nc3ccc(Cl)c(C(F)(F)F)c3)cc2)ccn1</smiles>

To further study the cytotoxic profile, the most potent compound $\mathbf{6 p}$ was selected for further evaluation of its inhibitory activity against a panel of human cancer cell lines. Interestingly, compound 6p showed broad-spectrum antiproliferative activities in vitro (Table 2). It had significant cytotoxicity against colon cancer cell lines HCT-116 and SW480, lung cancer cell line SPC-A1 and melanotic cancer cell line A375 with $\mathrm{IC}_{50}$ values $<10 \mu \mathrm{M}$. 
Table 2. Inhibition of cell proliferation by compound $\mathbf{6 p}$ and Sorafenib.

\begin{tabular}{lllllllll}
\hline \multirow{2}{*}{ Compound } & \multicolumn{7}{c}{$\mathbf{I C}_{\mathbf{5 0}}(\boldsymbol{\mu M M})^{\mathbf{a}}$} \\
\cline { 2 - 9 } & HepG2 & MCF-7 & HCT116 & SW480 & A549 & SPC-A1 & A375 & U87 \\
\hline 6p & 2.23 & 35.73 & 9.14 & 8.78 & 13.71 & 9.61 & 6.97 & 25.53 \\
Sorafenib & 16.30 & $>100$ & 10.09 & 40.65 & 13.15 & 18.60 & 17.96 & 62.19 \\
\hline \multicolumn{7}{c}{${ }^{a}$ Values are means of three independent experiments. }
\end{tabular}

Compound 6p was evaluated on six kinases at a concentration of $10 \mu \mathrm{M}$ (Table 3). It was found that 6p could selectively inhibit Aurora-B kinase to a significant level $(87 \%$ inhibition at $10 \mu \mathrm{M})$. This result provided a possible reason for its broad-spectrum antiproliferative activities.

Table 3. Kinase inhibitory assays of $\mathbf{6 p}$.

\begin{tabular}{cllllll}
\hline \multirow{2}{*}{ Compound } & \multicolumn{6}{c}{ \% inhibition at 10 $\boldsymbol{\mu M}$} \\
\cline { 2 - 7 } & Aurora-A & Aurora-B & Axl & Flt3 & KDR & PDGFR $\alpha$ \\
\hline 6p & -18 & 87 & -1 & -6 & 7 & 0 \\
\hline
\end{tabular}

\subsection{Molecular Docking Study}

In order to further investigate the interactions between compound $\mathbf{6 p}$ and the Aurora-B kinase, a docking study was performed using the Genetic Optimization for Ligand Docking (GOLD) 4.0 program. The crystal structure of Aurora-B (PDB Code: 4AF3) was used as the reference receptor.

According to the docking result, we can discern that two hydrogen bonds were formed between compound 6p and the Aurora-B kinase. As shown in Figure 4, the chlorine of the inhibitor formed one hydrogen bond with the residue Phe219 (2.452 $\left.\AA, 32.69^{\circ}\right)$, and another hydrogen bond was found between the residue Lys106 and the carbonyl group near the chlorine of the inhibitor $\left(2.178 \AA, 37.57^{\circ}\right)$. In addition, there was a $\pi-\pi$ conjugation interaction between the benzene ring group of compound $\mathbf{6 p}$ and the Phe 88 residue in the binding mode. The stable interactions between the inhibitor and Aurora-B kinase rationalize the obtained biological results.

Figure 4. The binding mode of compound 6p with ATP pocket of Aurora-B kinase obtained by molecular docking experiments (PDB code: 4AF3).

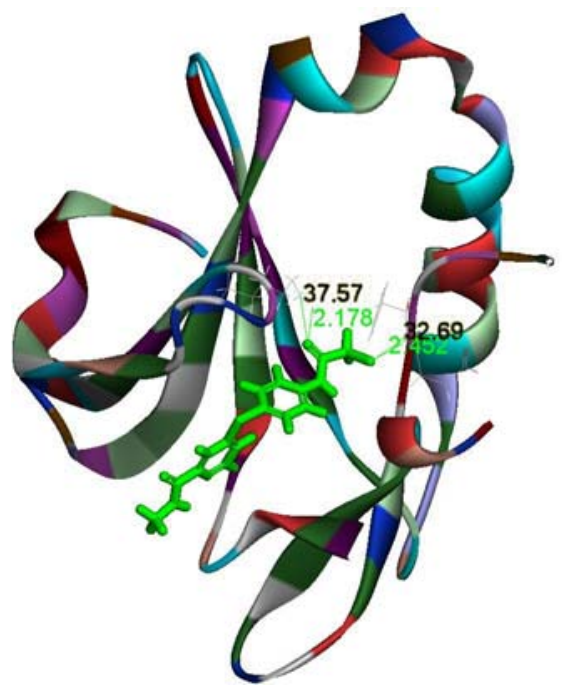




\section{Experimental}

\subsection{General}

The human cancer cell lines were purchased from the American Type Culture Collection (ATCC, Rockville, MD, USA). Dulbecco's modified Eagle medium (DMEM) and RPMI 1640 were purchased from Gibco (Grand Island, NY, USA). Fetal bovine serum (FBS) was purchased from Hyclone (Logan, UT, USA). Melting points were determined on a SGW X-4 microscopic melting point (Shanghai Precision \& Scientific Instrument Co., Ltd, Shanghai, China). ${ }^{1} \mathrm{H}-\mathrm{NMR}$ and ${ }^{13} \mathrm{C}-\mathrm{NMR}$ spectra were recorded on a Bruker Varian Unity Inova-400 (400/100 MHz) spectrometer using TMS as internal reference chemical. Shifts are expressed as $\delta$ values in ppm. Mass spectra (MS) were measured on a Q-TOF Premier mass spectrometer (Micromass, Manchester, UK) utilizing electrospray ionization (ESI).

\subsection{Preparation of 4-Chloropyridine-2-carbonyl Chloride Hydrochloride (4)}

4-Chloropyridine-2-carbonyl chloride hydrochloride was prepared according to the reported method. Thionyl chloride (198 g, $1.68 \mathrm{~mol})$ was added to a mixture of 2-picolinic acid (60 g, $0.48 \mathrm{~mol})$, sodium bromide $(8.08 \mathrm{~g}, 0.0785 \mathrm{~mol})$ and chlorobenzene $(84.8 \mathrm{~g})$. Then $2.0 \mathrm{M}$ methylamine solution in methanol was added to afford 4 as a white solid. Yield: 48 g, 58\%; m.p. 34.0-38.0 ${ }^{\circ} \mathrm{C}$; ${ }^{1} \mathrm{H}-\mathrm{NMR}$ (DMSO- $\left.d_{6}\right): \delta 3.04(\mathrm{~d}, J=5.2 \mathrm{~Hz}, 3 \mathrm{H}), 7.43(\mathrm{dd}, J=5.2,2.0 \mathrm{~Hz}, 1 \mathrm{H}), 7.98(\mathrm{~s}, 1 \mathrm{H}), 8.21$ (d, $J=1.6 \mathrm{~Hz}$, $1 \mathrm{H}), 8.44(\mathrm{~d}, J=5.2 \mathrm{~Hz}, 1 \mathrm{H})$; ESI-MS: $m / z 193.04(\mathrm{M}+\mathrm{Na})^{+}$.

\subsection{Preparation of 4-(4-Aminophenylthio)-N-methylcarboxamide (5)}

Potassium tert-butoxide $(4.04 \mathrm{~g}, 36.00 \mathrm{mmol})$ was added to a stirred solution of 4-aminothiophenol $(3.76 \mathrm{~g}, 30.00 \mathrm{mmol})$ in dry $N, N$-dimethylformamide $(58.68 \mathrm{~mL})$, and the reddish-brown mixture was stirred at room temperature for $3.5 \mathrm{~h}$. To the mixture was added $4(5.89 \mathrm{~g}, 34.50 \mathrm{mmol})$ and potassium carbonate $(25.44 \mathrm{~g}, 180.00 \mathrm{mmol})$, and then stirred at $85{ }^{\circ} \mathrm{C}$ under nitrogen for $15 \mathrm{~h}$. The mixture was cooled to room temperature and poured into the mixture of ethyl acetate $(200 \mathrm{~mL})$ and brine $(200 \mathrm{~mL})$. The aqueous layer was extracted with ethyl acetate $(150 \mathrm{~mL})$. The combined organic layers were washed with brine $(3 \times 400 \mathrm{~mL})$, dried over sodium sulfate, and concentrated to afford 5 as an orange solid. Yield: 6.76 g, 87\%; m.p. $112.8-115.1{ }^{\circ} \mathrm{C}$; ${ }^{1} \mathrm{H}-\mathrm{NMR}$ (DMSO- $d_{6}$ ): $\delta 2.81$ (q, $J=10.0 \mathrm{~Hz}, 3 \mathrm{H}$ ), $5.74(\mathrm{~s}, 2 \mathrm{H}), 6.68(\mathrm{~d}, J=8.4 \mathrm{~Hz}, 2 \mathrm{H}), 7.18(\mathrm{dd}, J=3.6,23.6 \mathrm{~Hz}, 3 \mathrm{H}), 7.49$ (s, 1H), 8.34 (d, $J=5.2 \mathrm{~Hz}$, 1H), $8.71(\mathrm{~d}, J=4.4 \mathrm{~Hz}, 1 \mathrm{H}) ;{ }^{13} \mathrm{C}-\mathrm{NMR}$ (DMSO- $\left.d_{6}\right): \delta 164.43,154.72,151.47,150.44,148.39,137.46$ (2C), 121.94, 117.37, 115.57 (2C), 110.60, 26.42; ESI-MS: $m / z 282.29(\mathrm{M}+\mathrm{Na})^{+}$.

\subsection{General Procedure for Preparing Compounds $\mathbf{1}$ and $\mathbf{6 a}-\mathbf{w}$}

Compound 5 (0.52 g, $2.00 \mathrm{mmol})$ and anhydrous potassium carbonate $(0.69 \mathrm{~g}, 5.00 \mathrm{mmol})$ were suspended in THF $(7.00 \mathrm{~mL})$, and then different substituted benzoyl chlorides or alkyl acyl chlorides ( $2.10 \mathrm{mmol}$ ) was added dropwise at $0-5{ }^{\circ} \mathrm{C}$. The mixture was stirred at room temperature for $2 \mathrm{~h}$ and poured into the mixture of ethyl acetate $(50 \mathrm{~mL})$ and brine $(50 \mathrm{~mL})$. The aqueous layer was back extracted with ethyl acetate $(3 \times 30 \mathrm{~mL})$. The combined organic layers were washed with brine $(3 \times 50 \mathrm{~mL})$, dried over sodium sulfate, and concentrated to afford compounds $\mathbf{6 a}-\mathbf{w}$. 
4-(4-Benzamidophenylthio)-N-methylpicolinamide (6a). Orange solid; yield: 91.87\%; m.p. 139.1-140.7 ${ }^{\circ} \mathrm{C}$; ${ }^{1} \mathrm{H}-\mathrm{NMR}\left(\mathrm{DMSO}-d_{6}\right): \delta 2.77(\mathrm{~d}, J=4.4 \mathrm{~Hz}, 3 \mathrm{H}), 7.27(\mathrm{dd}, J=2.0,5.2 \mathrm{~Hz}, 1 \mathrm{H}), 7.56(\mathrm{t}, J=7.4 \mathrm{~Hz}$, 2H), 7.61-7.64 (m, 4H), $8.02(\mathrm{q}, J=7.86 \mathrm{~Hz}, 4 \mathrm{H}), 8.42(\mathrm{~d}, J=5.2 \mathrm{~Hz}, 1 \mathrm{H}), 8.84(\mathrm{~d}, J=4.4 \mathrm{~Hz}, 1 \mathrm{H})$, $10.63(\mathrm{~s}, 1 \mathrm{H}) ;{ }^{13} \mathrm{C}-\mathrm{NMR}$ (DMSO- $\left.d_{6}\right): \delta 166.48,164.30,152.61,150.64,148.71,141.69,136.56(2 \mathrm{C})$, 135.19, 132.27, 128.91 (2C), 128.25 (2C), 122.61, 122.03 (2C), 121.84, 117.91, 26.44; ESI-MS: $\mathrm{m} / \mathrm{z}$ $364.44(\mathrm{M}+\mathrm{H})^{+}$.

4-(4-(3-Methoxybenzamido)phenylthio)-N-methylpicolinamide (6b). Orange solid; yield: 35.47\%; m.p. 60.1-63.4 ${ }^{\circ} \mathrm{C}$; ${ }^{1} \mathrm{H}-\mathrm{NMR}$ (DMSO- $d_{6}$ ): $\delta 2.77(\mathrm{~d}, J=4.8 \mathrm{~Hz}, 3 \mathrm{H}), 3.84(\mathrm{~d}, J=8.0 \mathrm{~Hz}, 3 \mathrm{H}$ ), 7.18 (dd, $J=2.4,8.0 \mathrm{~Hz}, 1 \mathrm{H}), 7.25(\mathrm{dd}, J=1.6,5.2 \mathrm{~Hz}, 1 \mathrm{H}), 7.45-7.63(\mathrm{~m}, 6 \mathrm{H}), 7.99(\mathrm{~d}, J=8.4 \mathrm{~Hz}, 2 \mathrm{H}), 8.40$ $(\mathrm{d}, J=5.6 \mathrm{~Hz}, 1 \mathrm{H}), 8.69(\mathrm{~d}, J=4.4 \mathrm{~Hz}, 1 \mathrm{H}), 10.48(\mathrm{~s}, 1 \mathrm{H}) ;{ }^{13} \mathrm{C}-\mathrm{NMR}\left(\mathrm{DMSO}-d_{6}\right): \delta 165.69,163.78$, 159.16, 152.11, 150.14, 148.18, 141.13, 136.09 (2C), 136.05, 129.57, 122.09, 121.57 (2C), 121.38, 119.94, 117.45, 117.39, 113.04, 55.31, 25.93; ESI-MS: $m / z$ 394.45 (M+H) .

4-(4-(4-Methoxybenzamido)phenylthio)-N-methylpicolinamide (6c). Orange solid; yield: 33.20\%; m.p. 155.2-158.7 ${ }^{\circ} \mathrm{C}$; ${ }^{1} \mathrm{H}-\mathrm{NMR}$ (DMSO- $\left.d_{6}\right): \delta 2.76(\mathrm{t}, J=5.8 \mathrm{~Hz}, 3 \mathrm{H}), 3.85(\mathrm{t}, J=9.8 \mathrm{~Hz}, 3 \mathrm{H}), 7.09(\mathrm{~d}$, $J=9.2 \mathrm{~Hz}, 2 \mathrm{H}), 7.25(\mathrm{dd}, J=2.0,5.2 \mathrm{~Hz}, 1 \mathrm{H}), 7.54(\mathrm{~d}, J=1.6 \mathrm{~Hz}, 1 \mathrm{H}), 7.61(\mathrm{~d}, J=8.4 \mathrm{~Hz}, 2 \mathrm{H}), 7.99$ $(\mathrm{q}, J=4.0 \mathrm{~Hz}, 4 \mathrm{H}), 8.40(\mathrm{~d}, J=4.8 \mathrm{~Hz}, 1 \mathrm{H}), 8.75(\mathrm{~d}, J=4.8 \mathrm{~Hz}, 1 \mathrm{H}), 10.40(\mathrm{~s}, 1 \mathrm{H}) ;{ }^{13} \mathrm{C}-\mathrm{NMR}$ (DMSO- $\left.d_{6}\right)$ : $\delta 165.77,164.29,162.58,152.69,150.65,148.71,141.91,136.55$ (2C), 130.24 (2C), 127.14, 122.58, $121.96(2 \mathrm{C}), 121.46,117.88,114.14(2 \mathrm{C}), 55.93,26.43$; ESI-MS: $m / z$ 394.43 (M+H) ${ }^{+}$.

4-(4-(2-Methoxybenzamido)phenylthio)-N-methylpicolinamide (6d). Orange solid; yield: 82.47\%; m.p. 122.4-125.1 ${ }^{\circ} \mathrm{C} ;{ }^{1} \mathrm{H}-\mathrm{NMR}\left(\mathrm{DMSO}-d_{6}\right): \delta 2.76(\mathrm{~d}, J=4.8 \mathrm{~Hz}, 3 \mathrm{H}), 3.91(\mathrm{~s}, 3 \mathrm{H}), 7.08(\mathrm{t}, J=7.6 \mathrm{~Hz}$, $1 \mathrm{H}), 7.20(\mathrm{~d}, J=8.4 \mathrm{~Hz}, 1 \mathrm{H}), 7.26(\mathrm{dd}, J=2.0,5.2 \mathrm{~Hz}, 1 \mathrm{H}), 7.51-7.54(\mathrm{~m}, 2 \mathrm{H}), 7.60-7.64(\mathrm{~m}, 3 \mathrm{H})$, $7.95(\mathrm{~d}, J=8.4 \mathrm{~Hz}, 2 \mathrm{H}), 8.41(\mathrm{~d}, J=5.6 \mathrm{~Hz}, 1 \mathrm{H}), 8.76(\mathrm{~d}, J=4.8 \mathrm{~Hz}, 1 \mathrm{H}), 10.46(\mathrm{~s}, 1 \mathrm{H}) ;{ }^{13} \mathrm{C}-\mathrm{NMR}$ (DMSO- $d_{6}$ ): $\delta 165.55,164.30,156.94,152.68,150.65,148.70,141.50,136.70$ (2C), 132.62, 130.07, 125.41, 122.57, 121.61, 121.50 (2C), 120.95, 117.88, 112.44, 56.34, 26.44; ESI-MS: $m / z$ 416.14 (M+Na) ${ }^{+}$

4-(4-(3,5-Dimethoxybenzamido)phenylthio)-N-methylpicolinamide (6e). Orange solid; yield: 32.13\%; m.p. 153.9-157.4 ${ }^{\circ} \mathrm{C} ;{ }^{1} \mathrm{H}-\mathrm{NMR}$ (DMSO-d $)$ ): $\delta 2.77(\mathrm{~d}, J=4.4 \mathrm{~Hz}, 3 \mathrm{H}), 3.84(\mathrm{~s}, 6 \mathrm{H}), 6.75(\mathrm{~s}, 1 \mathrm{H}), 7.12$ $(\mathrm{d}, J=2.0 \mathrm{~Hz}, 2 \mathrm{H}), 7.26(\mathrm{dd}, J=2.0,5.2 \mathrm{~Hz}, 1 \mathrm{H}), 7.54(\mathrm{~s}, 1 \mathrm{H}), 7.63(\mathrm{~d}, J=8.4 \mathrm{~Hz}, 2 \mathrm{H}), 8.00(\mathrm{~d}$, $J=8.4 \mathrm{~Hz}, 2 \mathrm{H}), 8.41(\mathrm{~d}, J=5.2 \mathrm{~Hz}, 1 \mathrm{H}), 8.76(\mathrm{~d}, J=4.8 \mathrm{~Hz}, 1 \mathrm{H}), 10.49(\mathrm{~s}, 1 \mathrm{H}) ;{ }^{13} \mathrm{C}-\mathrm{NMR}\left(\mathrm{DMSO}-d_{6}\right)$ : $\delta 165.50,163.71,160.36$ (2C), 152.27, 150.02, 148.09, 141.08, 136.69, 136.03 (2C), 122.10, 121.64 (2C), 121.39, 117.42, 105.72 (2C), 103.47, 55.48 (2C), 25.93; ESI-MS: $m / z ~ 424.20(\mathrm{M}+\mathrm{H})^{+}$.

4-(4-(2-Chlorobenzamido)phenylthio)-N-methylpicolinamide (6f). Orange solid; yield: 70.04\%; m.p. 191.3-193.5 ${ }^{\circ} \mathrm{C} ;{ }^{1} \mathrm{H}-\mathrm{NMR}$ (DMSO- $\left.d_{6}\right): \delta 2.76(\mathrm{~d}, J=5.2 \mathrm{~Hz}, 3 \mathrm{H}), 7.26(\mathrm{t}, J=2.8 \mathrm{~Hz}, 1 \mathrm{H}), 7.46-7.66$ $(\mathrm{m}, 7 \mathrm{H}), 7.93(\mathrm{~d}, J=8.4 \mathrm{~Hz}, 2 \mathrm{H}), 8.41(\mathrm{~d}, J=5.6 \mathrm{~Hz}, 1 \mathrm{H}), 8.76(\mathrm{~d}, J=4.8 \mathrm{~Hz}, 1 \mathrm{H}), 10.86(\mathrm{~s}, 1 \mathrm{H})$; ${ }^{13} \mathrm{C}-\mathrm{NMR}$ (DMSO- $d_{6}$ ): $\delta 165.28,163.80,152.04,150.14,148.22,140.79,136.63,136.25(2 \mathrm{C}), 131.26$, 129.92, 129.66, 128.96, 127.26, 122.13, 121.66, 120.92 (2C), 117.41, 25.95; ESI-MS: $m / z$ 398.40 (M+H) ${ }^{+}$

4-(4-(3-Fluorobenzamido)phenylthio)-N-methylpicolinamide (6g). Orange solid; yield: 71.66\%; m.p. 187.9-189.0 ${ }^{\circ} \mathrm{C} ;{ }^{1} \mathrm{H}-\mathrm{NMR}$ (DMSO- $\left.d_{6}\right): \delta 2.77(\mathrm{~d}, J=4.8 \mathrm{~Hz}, 3 \mathrm{H}), 7.27(\mathrm{~d}, J=5.2 \mathrm{~Hz}, 1 \mathrm{H}), 7.49$ (t, 
$J=8.4 \mathrm{~Hz}, 1 \mathrm{H}), 7.54(\mathrm{~s}, 1 \mathrm{H}), 7.60-7.65(\mathrm{~m}, 3 \mathrm{H}), 7.83(\mathrm{q}, J=7.7 \mathrm{~Hz}, 2 \mathrm{H}), 8.01(\mathrm{~d}, J=8.0 \mathrm{~Hz}, 2 \mathrm{H})$, $8.42(\mathrm{~d}, J=5.2 \mathrm{~Hz}, 1 \mathrm{H}), 8.76(\mathrm{~d}, J=4.4 \mathrm{~Hz}, 1 \mathrm{H}), 10.62(\mathrm{~s}, 1 \mathrm{H}) ;{ }^{13} \mathrm{C}-\mathrm{NMR}$ (DMSO- $\left.d_{6}\right): \delta 165.03$, 164.26, 163.60, 161.17, 152.57, 150.62, 148.66, 141.40, 137.41, 136.55 (2C), 131.03, 124.46, 122.62, 122.11 (2C), 117.94, 115.22, 114.99, 26.43; ESI-MS: $m / z 380.17(\mathrm{M}-\mathrm{H})^{+}$.

4-(4-(2,4-Dichlorobenzamido)phenylthio)-N-methylpicolinamide (6h). Orange solid; yield: 59.61\%; m.p. 227.3-229.7 ${ }^{\circ} \mathrm{C} ;{ }^{1} \mathrm{H}-\mathrm{NMR}$ (DMSO- $d_{6}$ ): $\delta 2.77$ (d, $\left.J=4.8 \mathrm{~Hz}, 3 \mathrm{H}\right), 7.26(\mathrm{dd}, J=2.0,5.2 \mathrm{~Hz}, 1 \mathrm{H}$ ), 7.55-7.64 (m, 5H), $7.79(\mathrm{~d}, J=1.6 \mathrm{~Hz}, 1 \mathrm{H}), 7.90(\mathrm{~d}, J=8.4 \mathrm{~Hz}, 2 \mathrm{H}), 8.41(\mathrm{~d}, J=5.2 \mathrm{~Hz}, 1 \mathrm{H}), 8.70(\mathrm{~d}$, $J=4.8 \mathrm{~Hz}, 1 \mathrm{H}), 10.84(\mathrm{~s}, 1 \mathrm{H}) ;{ }^{13} \mathrm{C}-\mathrm{NMR}\left(\mathrm{DMSO}-d_{6}\right): \delta 164.35,163.78,151.97,150.15,148.21$, 140.60, 136.27 (2C), 135.41, 135.07, 131.24, 130.38, 129.22, 127.47, 122.15, 121.88, 120.95 (2C), 117.41, 25.94; ESI-MS: $m / z 432.23(\mathrm{M}+\mathrm{H})^{+}$.

4-(4-(2,6-Difluorobenzamido)phenylthio)-N-methylpicolinamide (6i). Orange solid; yield: 64.15\%; m.p. 188.2-190.1 ${ }^{\circ} \mathrm{C} ;{ }^{1} \mathrm{H}-\mathrm{NMR}$ (DMSO- $\left.d_{6}\right): \delta 2.78(\mathrm{~d}, J=4.8 \mathrm{~Hz}, 3 \mathrm{H}), 7.24-7.32(\mathrm{~m}, 3 \mathrm{H}), 7.57$ (s, 1H), $7.60-7.66(\mathrm{~m}, 3 \mathrm{H}), 7.89$ (d, $J=8.4 \mathrm{~Hz}, 2 \mathrm{H}), 8.41$ (d, $J=5.2 \mathrm{~Hz}, 1 \mathrm{H}), 8.77$ (d, $J=4.8 \mathrm{~Hz}, 1 \mathrm{H}), 11.15$ $(\mathrm{s}, 1 \mathrm{H}) ;{ }^{13} \mathrm{C}-\mathrm{NMR}$ (DMSO- $\left.d_{6}\right): \delta 163.69,159.57,158.53,157.97,152.10,149.98,148.17,140.26$, 136.36 (2C), 132.32, 122.23, 122.16, 120.83 (2C), 117.54, 115.11, 112.18, 112.04, 25.95; ESI-MS: $m / z 422.12(\mathrm{M}+\mathrm{Na})^{+}$.

N-Methyl-4-(4-(2,3,4,5-tetrafluorobenzamido)phenylthio)picolinamide (6j). Orange solid; yield: 47.85\%; m.p. 183.2-185.7 ${ }^{\circ} \mathrm{C}$; ${ }^{1} \mathrm{H}-\mathrm{NMR}$ (DMSO- $d_{6}$ ): $\delta 2.76$ (d, $J=5.2 \mathrm{~Hz}, 3 \mathrm{H}$ ), 7.27 (dd, $J=2.0,5.2 \mathrm{~Hz}$, $1 \mathrm{H}), 7.52(\mathrm{~d}, J=2.0 \mathrm{~Hz}, 1 \mathrm{H}), 7.65(\mathrm{~d}, J=8.8 \mathrm{~Hz}, 2 \mathrm{H}), 7.83-7.87(\mathrm{~m}, 3 \mathrm{H}), 8.41(\mathrm{~d}, J=5.2 \mathrm{~Hz}, 1 \mathrm{H})$, $8.76(\mathrm{~d}, J=4.8 \mathrm{~Hz}, 1 \mathrm{H}), 10.95(\mathrm{~s}, 1 \mathrm{H}) ;{ }^{13} \mathrm{C}-\mathrm{NMR}$ (DMSO- $\left.d_{6}\right): \delta 164.02,160.25,158.71,156.87$, $152.15,150.40,148.45,140.49,136.53$ (2C), 132.55, 122.58, 122.41, 121.43 (2C), 117.65, 114.93, 112.34, 112.13, 26.16; ESI-MS: $m / z 458.04(\mathrm{M}+\mathrm{Na})^{+}$.

N-Methyl-4-(4-(3-(trifluoromethyl)benzamido)phenylthio)picolinamide (6k). Orange solid; yield: 71.09\%; m.p. 168.1-169.5 ${ }^{\circ} \mathrm{C}$; ${ }^{1} \mathrm{H}-\mathrm{NMR}$ (DMSO- $d_{6}$ ): $\delta 2.77$ (d, $J=4.8 \mathrm{~Hz}, 3 \mathrm{H}$ ), 7.26 (dd, $J=2.0,5.2 \mathrm{~Hz}$, 1H), $7.56(\mathrm{~s}, 1 \mathrm{H}), 7.65(\mathrm{~d}, J=8.8 \mathrm{~Hz}, 2 \mathrm{H}), 7.81(\mathrm{t}, J=7.8 \mathrm{~Hz}, 1 \mathrm{H}), 8.00(\mathrm{~d}, J=8.8 \mathrm{~Hz}, 3 \mathrm{H}), 8.28$ $(\mathrm{s}, 1 \mathrm{H}), 8.31(\mathrm{~d}, J=4.4 \mathrm{~Hz}, 1 \mathrm{H}), 8.41(\mathrm{~d}, J=5.2 \mathrm{~Hz}, 1 \mathrm{H}), 8.70(\mathrm{~d}, J=4.4 \mathrm{~Hz}, 1 \mathrm{H}), 10.73(\mathrm{~s}, 1 \mathrm{H})$; ${ }^{13} \mathrm{C}-\mathrm{NMR}$ (DMSO- $d_{6}$ ): $\delta 164.43,163.72,152.10,150.06,148.12,140.82,136.04$ (2C), 135.53, 131.89, 129.68, 128.26, 124.80, 124.36, 124.34, 122.12, 121.82, 121.70 (2C), 117.46, 25.91; ESI-MS: $\mathrm{m} / \mathrm{z}$ $432.35(\mathrm{M}+\mathrm{H})^{+}$.

N-Methyl-4-(4-(4-(trifluoromethyl)benzamido)phenylthio)picolinamide (61). Orange solid; yield: 56.73\%; m.p. 193.6-194.9 ${ }^{\circ} \mathrm{C}$; ${ }^{1} \mathrm{H}-\mathrm{NMR}$ (DMSO- $d_{6}$ ): $\delta 2.77$ (d, $J=4.4 \mathrm{~Hz}, 3 \mathrm{H}$ ), 7.27 (dd, $J=1.6,5.2 \mathrm{~Hz}$, 1H), 7.55 (s, 1H), 7.65 (d, $J=8.8 \mathrm{~Hz}, 2 \mathrm{H}), 7.95(\mathrm{~d}, J=8.0 \mathrm{~Hz}, 2 \mathrm{H}), 8.01$ (d, $J=8.8 \mathrm{~Hz}, 2 \mathrm{H}), 8.18$ (d, $J=8.0 \mathrm{~Hz}, 2 \mathrm{H}), 8.42(\mathrm{~d}, J=5.2 \mathrm{~Hz}, 1 \mathrm{H}), 8.76(\mathrm{~d}, J=4.8 \mathrm{~Hz}, 1 \mathrm{H}), 10.78(\mathrm{~s}, 1 \mathrm{H}) ;{ }^{13} \mathrm{C}-\mathrm{NMR}\left(\mathrm{DMSO}-d_{6}\right)$ : $\delta 164.79,163.77,152.00,150.16,148.19,140.83,138.47,136.07$ (2C), 128.68 (2C), 125.37, 125.35 (2C), 122.94, 122.12, 121.84, $121.62(2 \mathrm{C}), 117.43,25.91$; ESI-MS: $m / z 432.32(\mathrm{M}+\mathrm{H})^{+}$.

N-Methyl-4-(4-(2-(trifluoromethyl)benzamido)phenylthio)picolinamide (6m). Orange solid; yield: 71.22\%; m.p. 232.3-233.5 ${ }^{\circ} \mathrm{C} ;{ }^{1} \mathrm{H}-\mathrm{NMR}$ (DMSO-d $)$ ): $\delta 2.78$ (d, $\left.J=4.4 \mathrm{~Hz}, 3 \mathrm{H}\right), 7.27$ (d, $J=4.0 \mathrm{~Hz}$, 
1H), $7.56(\mathrm{~s}, 1 \mathrm{H}), 7.64(\mathrm{~d}, J=8.4 \mathrm{~Hz}, 2 \mathrm{H}), 7.73-7.85(\mathrm{~m}, 3 \mathrm{H}), 7.89$ (t, $J=6.8 \mathrm{~Hz}, 3 \mathrm{H}), 8.42(\mathrm{~d}, J=5.2 \mathrm{~Hz}$, $1 \mathrm{H}), 8.77(\mathrm{~d}, J=4.4 \mathrm{~Hz}, 1 \mathrm{H}), 10.92(\mathrm{~s}, 1 \mathrm{H}) ;{ }^{13} \mathrm{C}-\mathrm{NMR}$ (DMSO- $\left.d_{6}\right): \delta 165.90,163.80,151.99,150.16$, 148.23, 140.76, 136.24 (2C), 135.81, 132.62, 130.21, 128.56, 126.33, 124.63, 122.82, 122.14, 121.79, 120.96 (2C), 117.43, 25.93; ESI-MS: $m / z 430.00(\mathrm{M}-\mathrm{H})^{+}$.

N-Methyl-4-(4-(3-nitrobenzamido)phenylthio)picolinamide (6n). Orange solid; yield: 50.01\%; m.p. 200.4-201.1 ${ }^{\circ} \mathrm{C} ;{ }^{1} \mathrm{H}-\mathrm{NMR}$ (DMSO- $d_{6}$ ): $\delta 2.77$ (d, $J=4.8 \mathrm{~Hz}, 3 \mathrm{H}$ ), 7.26 (dd, $J=2.0,5.2 \mathrm{~Hz}, 1 \mathrm{H}$ ), 7.57 $(\mathrm{d}, J=1.6 \mathrm{~Hz}, 1 \mathrm{H}), 7.65(\mathrm{~d}, J=8.4 \mathrm{~Hz}, 2 \mathrm{H}), 7.86(\mathrm{t}, J=8.0 \mathrm{~Hz}, 1 \mathrm{H}), 8.05(\mathrm{~d}, J=8.4 \mathrm{~Hz}, 2 \mathrm{H}), 8.41(\mathrm{~d}$, $J=5.2 \mathrm{~Hz}, 1 \mathrm{H}), 8.46(\mathrm{dd}, J=1.6,8.4 \mathrm{~Hz}, 1 \mathrm{H}), 8.50(\mathrm{~d}, J=8.0 \mathrm{~Hz}, 1 \mathrm{H}), 8.70(\mathrm{~d}, J=5.2 \mathrm{~Hz}, 1 \mathrm{H}), 8.82$ (s, $1 \mathrm{H}), 11.02(\mathrm{~s}, 1 \mathrm{H}) ;{ }^{13} \mathrm{C}-\mathrm{NMR}$ (DMSO-d $)$ : $\delta 164.27$ (2C), 152.46, 150.65, 148.77, 148.18, 141.32, 136.49 (2C), 136.43, 134.89, 130.63, 126.79, 123.21, 122.64, 122.41, 122.36 (2C), 117.96, 26.44; ESI-MS: $m / z 409.19(\mathrm{M}+\mathrm{H})^{+}$.

N-Methyl-4-(4-(4-nitrobenzamido)phenylthio)picolinamide (6o). Orange solid; yield: 78.93\%; m.p. 215.9-216.6 ${ }^{\circ} \mathrm{C} ;{ }^{1} \mathrm{H}-\mathrm{NMR}\left(\mathrm{DMSO}-d_{6}\right): \delta 2.77(\mathrm{~d}, J=4.4 \mathrm{~Hz}, 3 \mathrm{H}), 7.27(\mathrm{~d}, J=5.2 \mathrm{~Hz}, 1 \mathrm{H}$ ), 7.55 (s, $1 \mathrm{H}), 7.66(\mathrm{~d}, J=8.4 \mathrm{~Hz}, 2 \mathrm{H}), 8.03(\mathrm{~d}, J=8.4 \mathrm{~Hz}, 2 \mathrm{H}), 8.23$ (d, $J=8.8 \mathrm{~Hz}, 2 \mathrm{H}), 8.41$ (t, $J=7.2 \mathrm{~Hz}$, $3 \mathrm{H}), 8.76(\mathrm{~d}, J=4.4 \mathrm{~Hz}, 1 \mathrm{H}), 10.93(\mathrm{~s}, 1 \mathrm{H}) ;{ }^{13} \mathrm{C}-\mathrm{NMR}$ (DMSO-d $)$ : $\delta 164.28,163.83,151.93,150.07$, $149.15,148.23,140.70,140.13,135.96$ (2C), 129.40 (2C), 123.43 (2C), 122.12, 122.02, 121.77 (2C), 117.45, 25.91; ESI-MS: $m / z$ 409.25 (M+H) ${ }^{+}$.

4-(4-Acetamidophenylthio)- $N$-methylpicolinamide (1). Orange solid; yield: 74.82\%; m.p. 181.7-184.1 ${ }^{\circ} \mathrm{C}$; ${ }^{1} \mathrm{H}-\mathrm{NMR}\left(\mathrm{DMSO}-d_{6}\right): \delta 2.10(\mathrm{~s}, 3 \mathrm{H}), 2.76(\mathrm{~d}, J=4.8 \mathrm{~Hz}, 3 \mathrm{H}), 7.23(\mathrm{dd}, J=2.0,5.2 \mathrm{~Hz}, 1 \mathrm{H}), 7.51(\mathrm{~d}$, $J=1.6 \mathrm{~Hz}, 1 \mathrm{H}), 7.56(\mathrm{~d}, J=8.8 \mathrm{~Hz}, 2 \mathrm{H}), 7.78(\mathrm{~d}, J=8.8 \mathrm{~Hz}, 2 \mathrm{H}), 8.39$ (d, $J=5.2 \mathrm{~Hz}, 1 \mathrm{H}), 8.74(\mathrm{~d}$, $J=4.8 \mathrm{~Hz}, 1 \mathrm{H}), 10.27(\mathrm{~s}, 1 \mathrm{H}) ;{ }^{13} \mathrm{C}-\mathrm{NMR}\left(\mathrm{DMSO}-d_{6}\right): \delta 168.77,163.77,152.24,150.10,148.14$, 141.25, $136.21(2 \mathrm{C}), 121.98,120.44,120.24(2 \mathrm{C}), 117.31,25.92,24.09$; ESI-MS: $m / z$ 324.11 (M+Na) ${ }^{+}$.

4-(4-(2-Chloroacetamido)phenylthio)-N-methylpicolinamide (6p). Orange solid; yield: 43.93\%; m.p. 203.1-205.7 ${ }^{\circ} \mathrm{C}$; ${ }^{1} \mathrm{H}-\mathrm{NMR}$ (DMSO- $d_{6}$ ): $\delta 2.77(\mathrm{t}, J=6.2 \mathrm{~Hz}, 3 \mathrm{H}), 4.35$ (s, 2H), 7.22 (dd, $J=2.0,4.8 \mathrm{~Hz}$, $1 \mathrm{H}), 7.53(\mathrm{~d}, J=2.0 \mathrm{~Hz}, 1 \mathrm{H}), 7.60(\mathrm{~d}, J=8.8 \mathrm{~Hz}, 2 \mathrm{H}), 7.83(\mathrm{~d}, J=8.8 \mathrm{~Hz}, 2 \mathrm{H}), 8.40(\mathrm{~d}, J=5.2 \mathrm{~Hz}$, $1 \mathrm{H}), 8.75(\mathrm{~d}, J=4.4 \mathrm{~Hz}, 1 \mathrm{H}), 10.90(\mathrm{~s}, 1 \mathrm{H}) ;{ }^{13} \mathrm{C}-\mathrm{NMR}$ (DMSO- $\left.d_{6}\right): \delta 165.67,164.22,152.63$, 150.51, 148.67, 141.10, 136.69 (2C), 122.54, 121.97, 121.15 (2C), 117.97, 44.01, 26.45; ESI-MS: $\mathrm{m} / \mathrm{z}$ $336.34(\mathrm{M}+\mathrm{H})^{+}$.

4-(4-(2,2-Dichloroacetamido)phenylthio)-N-methylpicolinamide (6q). Orange solid; yield: 70.01\%; m.p. 195.5-196.9 ${ }^{\circ} \mathrm{C} ;{ }^{1} \mathrm{H}-\mathrm{NMR}$ (DMSO- $\left.d_{6}\right): \delta 2.77$ (d, $\left.J=4.4 \mathrm{~Hz}, 3 \mathrm{H}\right), 6.66(\mathrm{~s}, 1 \mathrm{H}), 7.25$ (d, $J=4.4 \mathrm{~Hz}$, 1H), $7.54(\mathrm{~s}, 1 \mathrm{H}), 7.65(\mathrm{~d}, J=8.4 \mathrm{~Hz}, 2 \mathrm{H}), 7.82(\mathrm{~d}, J=8.0 \mathrm{~Hz}, 2 \mathrm{H}), 8.41(\mathrm{~d}, J=5.2 \mathrm{~Hz}, 1 \mathrm{H}), 8.75(\mathrm{~d}$, $J=5.2 \mathrm{~Hz}, 1 \mathrm{H}), 11.01(\mathrm{~s}, 1 \mathrm{H}) ;{ }^{13} \mathrm{C}-\mathrm{NMR}\left(\mathrm{DMSO}-d_{6}\right): \delta 163.73,162.07,151.68,150.15,148.26$, 139.48, 136.29 (2C), 122.89, 122.21, 121.16 (2C), 117.55, 67.22, 25.94; ESI-MS: m/z 368.02 (M-H) ${ }^{+}$

N-Methyl-4-(4-(2,2,2-trichloroacetamido)phenylthio)picolinamide (6r). Orange solid; yield: 28.15\%; m.p. 197.0-197.8 ${ }^{\circ} \mathrm{C} ;{ }^{1} \mathrm{H}-\mathrm{NMR}$ (DMSO- $d_{6}$ ): $\delta 2.77$ (d, $\left.J=4.8 \mathrm{~Hz}, 3 \mathrm{H}\right), 7.27$ (dd, $J=1.6,5.2 \mathrm{~Hz}, 1 \mathrm{H}$ ), $7.55(\mathrm{~d}, J=1.2 \mathrm{~Hz}, 1 \mathrm{H}), 7.67(\mathrm{~d}, J=8.4 \mathrm{~Hz}, 2 \mathrm{H}), 7.90(\mathrm{~d}, J=8.4 \mathrm{~Hz}, 2 \mathrm{H}), 8.42(\mathrm{~d}, J=5.2 \mathrm{~Hz}, 1 \mathrm{H})$, $8.76(\mathrm{~d}, J=4.8 \mathrm{~Hz}, 1 \mathrm{H}), 11.15(\mathrm{~s}, 1 \mathrm{H}) ;{ }^{13} \mathrm{C}-\mathrm{NMR}$ (DMSO- $\left.d_{6}\right): \delta 164.28,160.34,152.00,150.63$, 
148.80, 139.57, 136.46 (2C), 124.38, 123.08 (2C), 122.84, 118.14, 93.31, 26.44; ESI-MS: $m / z$ $401.97(\mathrm{M}-\mathrm{H})^{+}$.

4-(4-(3-Chloropropanamido)phenylthio)-N-methylpicolinamide (6s). Orange solid; yield: 58.10\%; m.p. 145.0-147.2 ${ }^{\circ} \mathrm{C}$; ${ }^{1} \mathrm{H}-\mathrm{NMR}$ (DMSO-d $)_{\text {) }} \delta 2.77$ (t, $J=6.4 \mathrm{~Hz}, 3 \mathrm{H}$ ), 2.88 (t, $J=6.2 \mathrm{~Hz}, 2 \mathrm{H}$ ), 3.90 (t, $J=6.4 \mathrm{~Hz}, 2 \mathrm{H}), 7.21(\mathrm{dd}, J=2.0,5.2 \mathrm{~Hz}, 1 \mathrm{H}), 7.53(\mathrm{~d}, J=1.2 \mathrm{~Hz}, 1 \mathrm{H}), 7.57(\mathrm{~d}, J=8.4 \mathrm{~Hz}, 2 \mathrm{H}), 7.80$ $(\mathrm{d}, J=8.8 \mathrm{~Hz}, 2 \mathrm{H}), 8.39(\mathrm{~d}, J=5.6 \mathrm{~Hz}, 1 \mathrm{H}), 8.68(\mathrm{~d}, J=4.4 \mathrm{~Hz}, 1 \mathrm{H}), 10.35(\mathrm{~s}, 1 \mathrm{H}) ;{ }^{13} \mathrm{C}-\mathrm{NMR}$ (DMSO- $d_{6}$ ): $\delta 168.96,164.26,152.64,150.61,148.67,141.37,136.77$ (2C), 122.52, 121.44, 120.90 (2C), 117.86, 60.21, 26.42, 14.52; ESI-MS: $m / z 372.19(\mathrm{M}+\mathrm{Na})^{+}$.

4-(4-(4-Chlorobutanamido)phenylthio)-N-methylpicolinamide (6t). Orange solid; yield: 76.45\%; m.p. 106.4-108.9 ${ }^{\circ} \mathrm{C} ;{ }^{1} \mathrm{H}-\mathrm{NMR}$ (DMSO- $\left.d_{6}\right): \delta 2.03-2.10(\mathrm{~m}, 2 \mathrm{H}), 2.54(\mathrm{q}, J=7.6 \mathrm{~Hz}, 2 \mathrm{H}), 2.76(\mathrm{~d}, J=5.2 \mathrm{~Hz}$, $3 \mathrm{H}), 3.72(\mathrm{t}, J=6.4 \mathrm{~Hz}, 2 \mathrm{H}), 7.23(\mathrm{~d}, J=4.0 \mathrm{~Hz}, 1 \mathrm{H}), 7.51(\mathrm{~s}, 1 \mathrm{H}), 7.56(\mathrm{~d}, J=8.8 \mathrm{~Hz}, 2 \mathrm{H}), 7.80(\mathrm{~d}$, $J=8.4 \mathrm{~Hz}, 2 \mathrm{H}), 8.40(\mathrm{~d}, J=5.2 \mathrm{~Hz}, 1 \mathrm{H}), 8.75(\mathrm{~d}, J=4.8 \mathrm{~Hz}, 1 \mathrm{H}), 10.33(\mathrm{~s}, 1 \mathrm{H}) ;{ }^{13} \mathrm{C}-\mathrm{NMR}\left(\mathrm{DMSO}-d_{6}\right)$ : $\delta 170.71,163.53,152.83,149.67,147.84,141.22,136.62,136.20$ (2C), 122.01, 120.37 (2C), 117.43, 44.94, 33.47, 27.76, 25.95; ESI-MS: $m / z 398.05(\mathrm{M}+\mathrm{Cl})^{-}$.

N-Methyl-4-(4-propionamidophenylthio)picolinamide (6u). Orange solid; yield: 53.49\%; m.p. 184.6-187.0 ${ }^{\circ} \mathrm{C}$; ${ }^{1} \mathrm{H}-\mathrm{NMR}\left(\mathrm{DMSO}-d_{6}\right): \delta 1.11(\mathrm{t}, J=7.6 \mathrm{~Hz}, 3 \mathrm{H}), 2.38(\mathrm{q}, J=7.6 \mathrm{~Hz}, 2 \mathrm{H}), 2.76(\mathrm{~d}$, $J=4.8 \mathrm{~Hz}, 3 \mathrm{H}), 7.23(\mathrm{dd}, J=1.6,5.2 \mathrm{~Hz}, 1 \mathrm{H}), 7.50(\mathrm{~s}, 1 \mathrm{H}), 7.56(\mathrm{~d}, J=8.4 \mathrm{~Hz}, 2 \mathrm{H}), 7.80(\mathrm{~d}, J=8.4 \mathrm{~Hz}$, $2 \mathrm{H}), 8.39(\mathrm{~d}, J=5.2 \mathrm{~Hz}, 1 \mathrm{H}), 8.75(\mathrm{~d}, J=4.4 \mathrm{~Hz}, 1 \mathrm{H}), 10.20(\mathrm{~s}, 1 \mathrm{H}) ;{ }^{13} \mathrm{C}-\mathrm{NMR}\left(\mathrm{DMSO}-d_{6}\right): \delta 172.73$, $164.05,152.57,150.35,148.38,141.61,136.50$ (2C), 122.22, 120.53 (2C), 117.55, 29.90, 26.21, 26.18, 9.77; ESI-MS: $m / z 314.10(\mathrm{M}-\mathrm{H})^{+}$.

4-(4-Butyramidophenylthio)-N-methylpicolinamide (6v). Orange solid; yield: 84.96\%; m.p. 144.8-147.0 ${ }^{\circ} \mathrm{C} ;{ }^{1} \mathrm{H}-\mathrm{NMR}$ (DMSO- $d_{6}$ ): $\delta 0.94(\mathrm{t}, J=7.4 \mathrm{~Hz}, 3 \mathrm{H}), 1.59-1.67$ (m, 2H), 2.34 (t, $J=7.4 \mathrm{~Hz}$, 2H), $2.77(\mathrm{~d}, J=4.8 \mathrm{~Hz}, 3 \mathrm{H}), 7.23(\mathrm{~d}, J=4.8 \mathrm{~Hz}, 1 \mathrm{H}), 7.51(\mathrm{~s}, 1 \mathrm{H}), 7.56(\mathrm{~d}, J=8.0 \mathrm{~Hz}, 2 \mathrm{H}), 7.80(\mathrm{~d}$, $J=8.4 \mathrm{~Hz}, 2 \mathrm{H}), 8.39(\mathrm{~d}, J=5.2 \mathrm{~Hz}, 1 \mathrm{H}), 8.74(\mathrm{~d}, J=4.8 \mathrm{~Hz}, 1 \mathrm{H}), 10.21(\mathrm{~s}, 1 \mathrm{H}) ;{ }^{13} \mathrm{C}-\mathrm{NMR}\left(\mathrm{DMSO}-d_{6}\right)$ : $\delta$ 171.61, 163.77, 152.26, 150.10, 148.13, 141.27, 136.20(2C), 121.97, 120.37(2C), 120.30, 117.31, 38.40, 25.91, 18.45, 13.57; ESI-MS: $m / z 328.12(\mathrm{M}-\mathrm{H})^{+}$.

N-Methyl-4-(4-pivalamidophenylthio)picolinamide (6w). Orange solid; yield: 51.57\%; m.p. 108.5-110.5 ${ }^{\circ} \mathrm{C} ;{ }^{1} \mathrm{H}-\mathrm{NMR}$ (DMSO- $d_{6}$ ): $\delta 1.26(\mathrm{~s}, 9 \mathrm{H}), 2.76(\mathrm{~d}, J=4.8 \mathrm{~Hz}, 3 \mathrm{H}), 7.24(\mathrm{~d}, J=5.2 \mathrm{~Hz}$, $1 \mathrm{H}), 7.51(\mathrm{~s}, 1 \mathrm{H}), 7.56(\mathrm{~d}, J=8.4 \mathrm{~Hz}, 2 \mathrm{H}), 7.89$ (d, $J=8.4 \mathrm{~Hz}, 2 \mathrm{H}), 8.40(\mathrm{~d}, J=5.2 \mathrm{~Hz}, 1 \mathrm{H}), 8.75(\mathrm{~d}$, $J=4.8 \mathrm{~Hz}, 1 \mathrm{H}), 9.50(\mathrm{~s}, 1 \mathrm{H}) ;{ }^{13} \mathrm{C}-\mathrm{NMR}\left(\mathrm{DMSO}-d_{6}\right): \delta 177.35,164.28,152.76,150.62,148.64,141.92$, 136.42 (2C), 122.53, $121.82(2 \mathrm{C}), 121.12,117.83,27.55$ (4C), 26.42; ESI-MS: $m / z$ 366.23 (M+Na) ${ }^{+}$.

\subsection{Cell Culture}

Cell lines including HepG2, A375 and U87 were maintained in Dulbecco's modified Eagle medium (DMEM) containing 10\% fetal bovine serum (FBS), penicillin (100 U/mL) and streptomycin (10 mg/L). Cell lines including HCT116, MCF-7, SPC-A1, A549 and SW480, were maintained in RPMI 1640 containing $10 \%$ fetal bovine serum (FBS), penicillin $(100 \mathrm{U} / \mathrm{mL})$ and streptomycin $(10 \mathrm{mg} / \mathrm{L})$. Cells were grown in a $5 \% \mathrm{CO}_{2}$ incubator at $37{ }^{\circ} \mathrm{C}$. 


\subsection{Cell Proliferation Assay (MTT Assay)}

Cells $\left(3 \times 10^{3} /\right.$ well) were seeded in 96-well plates and cultured for $24 \mathrm{~h}$, followed by treatment with the compounds for $48 \mathrm{~h}$. Ten microliters of $10 \mathrm{mg} / \mathrm{mL}$ MTT was added per well and incubated for another $2.5 \mathrm{~h}$ at $37^{\circ} \mathrm{C}$. Then the supernatant fluid was removed and $150 \mu \mathrm{L} /$ well DMSO was added for 15-20 min. The absorbance (OD) of each well was measured at $570 \mathrm{~nm}$ using an ELISA reader (Thermo). The effect of compounds on tumor cells viability was expressed by $\mathrm{IC}_{50}$ of each cell line.

\subsection{Kinase Inhibitory Assay}

In vitro kinase inhibitory assays were performed against recombinant human Aurora-B kinase at the $\mathrm{Km}$ of ATP $(15 \mu \mathrm{M})$ and at a fixed concentration of $10 \mu \mathrm{M}$ of test compound. Each assay was repeated twice. All the inhibitory assays against Aurora-B were carried out through kinase profiling services provided by Millipore (America), in which radiometric protein kinase assays were used.

\section{Conclusions}

In conclusion, a series of novel $N$-methylpicolinamide-4-thiol derivatives has been synthesized and evaluated on human cancer cell lines. Among them, compound 6p was found to be the most potent, displaying broad-spectrum in vitro antiproliferative activities. The results of the MTT assay showed that compound $\mathbf{6 p}$ had significant cytotoxicity against liver cancer cell line HepG2, colon cancer cell lines HCT-116 and SW480, lung cancer cell line SPC-A1 and melanotic cancer cell line A375 with $\mathrm{IC}_{50}$ values $<10 \mu \mathrm{M}$. All these antiproliferative activities were better than those of the reference compound sorafenib. The advanced kinase inhibitory assays, which were performed on six kinases at a concentration of $10 \mu \mathrm{M}$, indicated that $\mathbf{6 p}$ could selectively inhibit Aurora-B kinase. A molecular docking study showed the stable interactions of $\mathbf{6 p}$ with the Aurora-B kinase, which rationalized the obtained biological results. Our ongoing work aimed at researching the advanced mechanism of action and explore the efficacy of compound $\mathbf{6 p}$ in a range of in vivo models, will be the subjects of future reports.

\section{Acknowledgments}

This work was supported by National Science and Technology Major Project of China (2009ZX09103-132). The authors are grateful to the State Key Laboratory of Biotherapy and Cancer Center for supporting this research project.

\section{References and Notes}

1. Glover, D.M.; Leibowitz, M.H.; McLean, D.A.; Parry, H. Mutations in aurora prevent centrosome separation separation leading to the formation of monopolar spindles. Cell 1995, 81, 95-105.

2. Carmena, M.; Earnshaw, W.C. The cellular geography of aurora kinases. Nat. Rev. Mol. Cell Biol. 2003, 4, 842-854.

3. Sen, S.; Zhou, H.; White, R.A. A putative serine/threonine kinase encoding gene BTAK on chromosome 20q13 is amplified and overexpressed in human breast cancer cell lines. Oncogene 1997, 14, 2195-2200. 
4. Bischoff, J.R.; Anderson, L.; Zhu, Y.; Mossie, K.; Ng, L.; Souza, B.; Schryver, B.; Flanagan, P.; Clairvoyant, F.; Ginther, C.; et al. A homologue of Drosophila aurora kinase is oncogenic and amplified in human colorectal cancers. EMBO J. 1998, 17, 3052-3065.

5. Katayama, H.; Ota, T.; Jisaki, F.; Ueda, Y.; Tanaka, T.; Odashima, S.; Suzuki, F.; Terada, Y.; Tatsuka, M. Mitotic kinase expression and colorectal cancer progression. J. Natl. Cancer Inst. 1999, 91, 1160-1162.

6. Kamada, K.; Yamada, Y.; Hirao, T.; Fujimoto, H.; Takahama, Y.; Ueno, M.; Takayama, T.; Naito, A.; Hirao, S.; Nakajima, Y. Amplification/overexpression of Aurora-A in human gastric carcinoma: Potential role in differentiated type gastric carcinogensis. Oncol. Rep. 2004, 12, 593-599.

7. Jackson, J.R.; Patrick, D.R.; Dar, M.M.; Huang, P.S. Targeted anti-mitotic therapies: Can we improve on tubulin agents. Nat. Rev. Cancer 2007, 7, 107-117.

8. Cheetham, G.M.T.; Charlton, P.A.; Golec, J.M.C.; Pollard, J.R. Structural basis for potent inhibition of the Aurora kinases and a T315I multi-drug resistant mutant form of Abl kinase by VX-680. Cancer Lett. 2007, 251, 323-329.

9. Harrington, E.A.; Bebbington, D.; Moore, J.; Rasmussen, R.K.; Ajose-Adeogun, A.O.; Nakayama, T.; Graham, J.A.; Demur, C.; Hercend, T.; Diu-Hercend, A.; et al. VX-680, a potent and selective small-molecule inhibitor of the Aurora kinases, suppresses tumor growth in vivo. Nat. Med. 2004, 10, 262-267.

10. Manfredi, M.G.; Ecsedy, J.A.; Meetze, K.A.; Balani, S.K.; Burenkova, O.; Chen, W.; Galvin, K.M.; Hoar, K.M.; Huck, J.J.; LeRoy, P.J.; et al. Antitumor activity of MLN8054, an orally active small-molecule inhibitor of Aurora A kinase. Proc. Natl. Acad. Sci. USA 2007, 104, 4106-4111.

11. Carpinelli, P.; Ceruti, R.; Giorgini, M.L.; Cappella, P.; Gianellini, L.; Croci, V.; Degrassi, A.; Texido, G.; Rocchetti, M.; Vianello, P.; et al. PHA-739358, a potent inhibitor of Aurora kinases with a selective target inhibition profile relevant to cancer. Mol. Cancer Ther. 2007, 6, 3158-3168.

12. Mortlock, A.A.; Foote, K.M.; Heron, N.M.; Jung, F.H.; Pasquet, G.; Lohmann, J.J.M.; Warin, N.; Renaud, F.; de Savi, C.; Roberts, N.J.; et al. Discovery, Synthesis, and in vivo activity of a new class of pyrazoloquinazolines as selective inhibitors of Aurora B kinase. J. Med. Chem. 2007, 50, 2213-2224.

13. Zhao, C.Y.; Chen, L.J.; Xu, X.; Luo, X.Y.; Ji, Y.F. Adeno-associated virus-mediated pancreatic and duodenal homeobox gene 1 delivery induced insulin-producing cells in livers of diabetic rats. Chin. J. Clin. Pharm. 2007, 38, 614-619.

14. Donald, B.; Jacques, D.; Reina, N.; Bernd, R.; Mary, K.M.; Robert, S. A Scaleable synthesis of BAY 43-9006: A potent Raf kinase inhibitor for the treatment of cancer. Org. Process Res. Dev. 2002, 6, 777-781.

15. Chen, A.; Huang, H.X.; Song, S.J.; Chen, X.Q. Synthesis of Imatinib Mesylate. Fine Spec. Chem. 2007, 8, 23-27.

Sample Availability: Not available.

(C) 2012 by the authors; licensee MDPI, Basel, Switzerland. This article is an open access article distributed under the terms and conditions of the Creative Commons Attribution license (http://creativecommons.org/licenses/by/3.0/). 